\title{
Alignment strategies for the entrainment of music and movement rhythms
}

\author{
Bart Moens and Marc Leman \\ Institute for Psychoacoustics and Electronic Music, Department of Musicology, Ghent University, Ghent, Belgium \\ Address for correspondence: Marc Leman, Institute for Psychoacoustics and Electronic Music (IPEM), Department of \\ Musicology, Ghent University, Sint-Pietersnieuwstraat 41, Technicum Blok 2, 9000 Ghent, Belgium. marc.leman@ugent.be
}

\begin{abstract}
Theories of entrainment assume that spontaneous entrainment emerges from dynamic laws that operate via mediators on interactions, whereby entrainment is facilitated if certain conditions are fulfilled. In this study, we show that mediators can be built that affect the entrainment of human locomotion to music. More specifically, we built D-Jogger, a music player that functions as a mediator between music and locomotion rhythms. The D-Jogger makes it possible to manipulate the timing differences between salient moments of the rhythms (beats and footfalls) through the manipulation of the musical period and phase, which affect the condition in which entrainment functions. We conducted several experiments to explore different strategies for manipulating the entrainment of locomotion and music. The results of these experiments showed that spontaneous entrainment can be manipulated, thereby suggesting different strategies on how to embark. The findings furthermore suggest a distinction among different modalities of entrainment: finding the beat (the most difficult part of entrainment), keeping the beat (easier, as a temporal scheme has been established), and being in phase (no entrainment is needed because the music is always adapted to the human rhythm). This study points to a new avenue of research on entrainment and opens new perspectives for the neuroscience of music.
\end{abstract}

Keywords: entrainment; synchronization; music; walking; D-Jogger

\section{Introduction}

Entrainment is the gradual fall into synchronism of two rhythms, such as when a locomotion pattern gradually falls into synchronization with a musical pattern. Entrainment is known to rely on continuous phase-correction adjustments, ${ }^{1}$ which can be measured with respect to the time difference between salient points of the rhythms (termed the relative phase). A salient point in walking, for example, occurs when the foot touches the ground (the footfall), whereas in music it is characterized by the beat.

Owing to entrainment the time difference of the footfall and the beat gradually diminishes, and it comes close to zero when a stable synchronization between the two rhythms is reached. The above mechanism is well known and has been mathematically described with respect to an attraction dynamic. $^{2,3}$ The models show that spontaneous entrainment emerges from dynamic laws that operate via mediators on interactions, whereby entrainment is facilitated if certain conditions are fulfilled. ${ }^{4}$

However, to date, little attention has been paid to the setup in which rhythms influence each other. A simple setup occurs in the classical synchronizationcontinuation paradigm in which subjects are asked to tap along with a metronome tick having a fixed intertick interval, until the moment where the metronome is stopped and the subjects continue to tap along on their own. ${ }^{1}$ The steady (nonadaptive) intertick interval of the metronome is a particular setup, and it places particular constraints on entrainment. Another setup occurs in interpersonal entrainment or in person-machine entrainment, as in pairs of people walking side by side ${ }^{5}$ or in interlimb coordination when only visual coupling is available. ${ }^{6}$ In this setup, entrainment is based on a mutual adjustment. Miyake ${ }^{7}$ showed that a human 


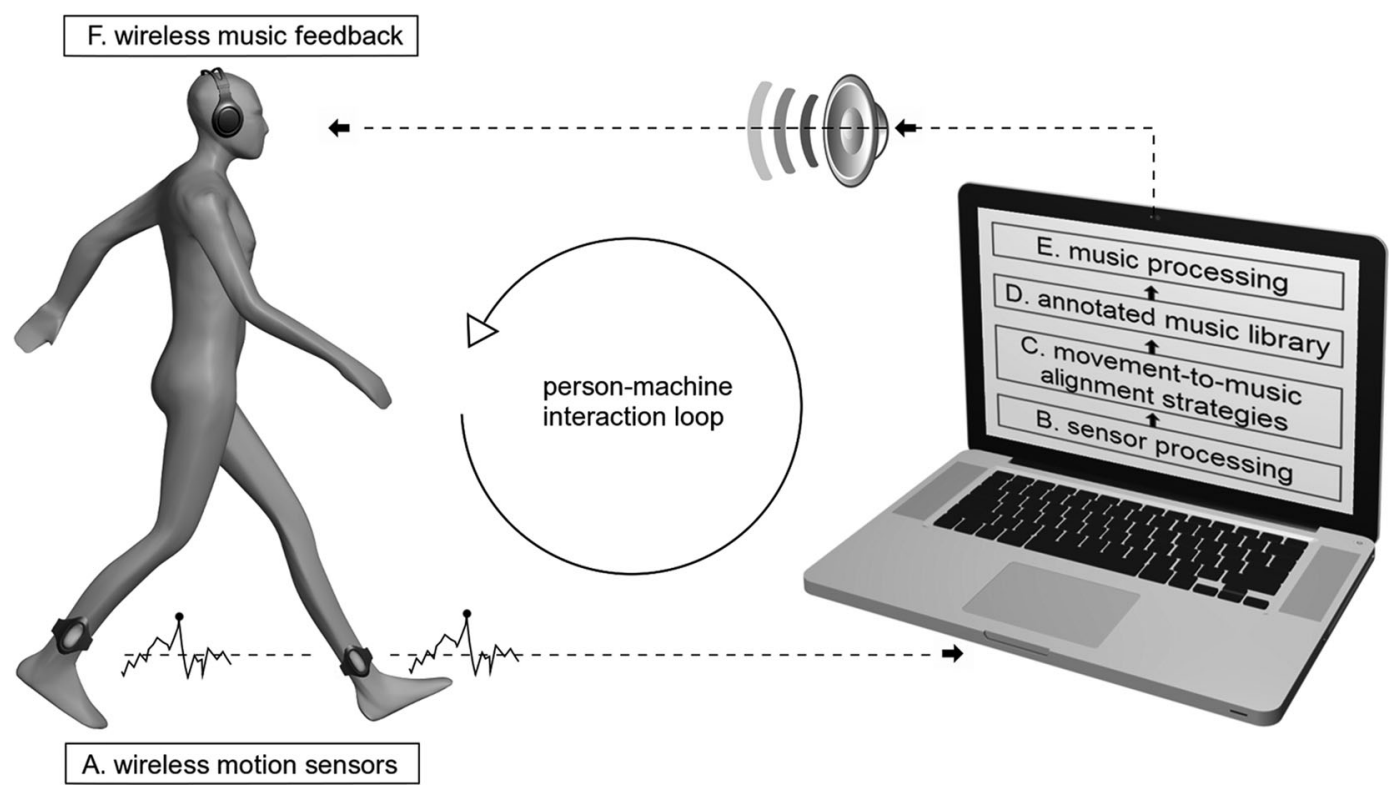

Figure 1. Smart music player: person-machine interaction loop and the main components involved.

and a robot can adapt mutually and maintain stable synchronization using a metronome as cue. Fairhurst et al. ${ }^{8}$ found that synchrony was more easily established when the virtual partner's adaptation rate was slow. These and other setups are examples of entrainment mediators that determine the influence of rhythms on each other.

The goal of this paper is to draw attention to the way in which these mediators can be manipulated to affect entrainment. Such manipulations would be called alignment strategies because they regulate how the timing framework of the locomotion and music rhythms matches. We present four empirically tested alignment strategies identifying how musical and human rhythms are related to each other and discuss the effect of these strategies on spontaneous entrainment.

The structure of the paper is presented as follows. First, we introduce the basic concepts of an interactive music player that facilitates a manipulation of parameters that influence the stability of the musical rhythm. We then go on to examine the four different strategies in greater depth to find the alignment of two rhythms. Finally, we discuss the major observations in the light of a refined concept of entrainment.

\section{D-Jogger technology}

D-Jogger is a technology that allows the manipulation of the period and phase of a song played on a music player. ${ }^{9}$ The technology was developed first and foremost for walking and running applications, but it can also be coupled with biological rhythms such as breathing and heart rate. After a playlist of songs of differing tempi has been introduced, $\mathrm{D}$-Jogger is able to increase or decrease the tempo of a song to match an external rhythm, enabling the music to fall in synchrony with the progression of the movements; it successfully makes incremental adjustments to achieve an alignment of both rhythms. If a subject suddenly changes tempo, from running to walking, for example, so that the difference in tempo exceeds the range of adaptation of the initial tempo, then D-Jogger selects another song that sits within the range of the person's new tempo. Even more interesting, perhaps, is that the D-Jogger can also change the phase of a song so that it begins simultaneously with the footfall. The phase can also be adapted after a pause in motion. Figure 1 shows the person-machine interaction and the required components. The three basic components of the D-Jogger technology are discussed later. 
Table 1. Overview of the four alignment strategies

\begin{tabular}{|c|c|c|c|c|}
\hline Alignment strategy & 1 & 2 & 3 & 4 \\
\hline Period adaption & Adaptive & Fixed & Adaptive & Adaptive \\
\hline Phase adaption & Random & Random & Fixed starting phase & Adaptive to $0^{\circ}$ \\
\hline Valid participants $(n)$ & 119 & 100 & 10 & 12 \\
\hline Participants' age & $22 \pm 12$ & $21 \pm 1.5$ & $21 \pm 2$ & $24 \pm 3$ \\
\hline Setup & PC & Mobile & PC & PC \\
\hline Walking area & Treadmill & Outdoor & Treadmill & Treadmill \\
\hline Stimuli & Pop selection & Pop selection & Pop selection & Motivational pop \\
\hline Tasks & Any tempo & Walk & Any tempo & Run \\
\hline Duration & Voluntary & 2-km walk & $15 \min$ & $30 \mathrm{~min}$ \\
\hline Recorded valid steps & 52,050 & 29,419 & 19,875 & 104,334 \\
\hline Mean angle $\Phi$ & $5.6^{\circ}$ & $4.7^{\circ}$ & $4.7^{\circ}$ & $-0.14^{\circ}$ \\
\hline High limit (95\% CI) & $7.6^{\circ}$ & $6.5^{\circ}$ & $5.4^{\circ}$ & $0.004^{\circ}$ \\
\hline Low limit (95\% CI) & $3.5^{\circ}$ & $3.0^{\circ}$ & $4.1^{\circ}$ & $-0.29^{\circ}$ \\
\hline Resultant vector length $|R|$ & 0.17 & 0.27 & 0.73 & 0.92 \\
\hline Circular variance $(C V)$ & 0.83 & 0.73 & 0.27 & 0.08 \\
\hline Angular deviation $(s)$ & 1.30 & 1.2 & 0.73 & 0.4 \\
\hline Circular skewness $(b)$ & 0.015 & 0.0095 & 0.042 & 0.024 \\
\hline Circular kurtosis $(k)$ & 0.066 & 0.13 & 0.52 & 0.84 \\
\hline \multirow[t]{2}{*}{ Rayleigh test } & $Z_{1487.7}$ & $Z_{1907.8}$ & $Z_{10721.3}$ & $Z_{88674}$ \\
\hline & $P<0.001$ & $P<0.001$ & $P<0.001$ & $P<0.001$ \\
\hline
\end{tabular}

Note: Shown is the alignment algorithm, the methodological setting of the experiment, the resulting distributions using circular statistics, ${ }^{25}$ and finally, the results of the Rayleigh test. ${ }^{26}$ The described distributions indicate the distribution of relative phase angles of all the recorded steps. Most notable is the resultant vector length $I R I$, which gives an indication of the synchronization: a higher value shows more clustered relative phase angles around the mean. Finally, the Rayleigh test is used to test circular uniformity; here, it assesses whether participants performed above chance, that is, whether the alignment algorithm has a bearing on the participant's behavior.

\section{Movement processing}

The time instances of the marker for locomotion (herein referred to as the footfall) are captured using a wide range of sensors (e.g., 3D accelerometers, gyroscopes, and pressure sensors can be used; Fig. 1A) and streamed wirelessly to a computer where gait frequency (in steps per minute (SPM)) and gait phase are extracted (Fig. 1B), using empirically verified algorithms. ${ }^{10-12}$

\section{Music processing}

The system draws from a music database containing a selection of songs and metadata that consist of the average beats per minute (BPM) and the timings of the beat (phase information) (Fig. 1D). These time instances are extracted offline, using dedicated beat-extraction software such as BeatRoot. ${ }^{13}$ In addition, a phase vocoder is used to stretch the music in real time (Fig. 1E), using a combination of frequency- and time-domain methods based on ZPlanes Elastique Pitch audio technology. ${ }^{14}$ This allows for a change of tempo (which is acceptable for music deformation of up to $+10 \%$ and $-10 \%$ ) and/or phase of a song without changing its pitch.

\section{Alignment strategies}

The control of the phase vocoder is managed by a type of strategy that aligns the relative phase of the footfall and the beat (Fig. 1C). In what follows are four alignment strategies: (1) the song starts in tempo but not in phase. During the song, the music period is adapted to the walking period; (2) the song starts in tempo but not in phase. During the song, the music period is kept fixed and the subject entrains to the phase of the song; (3) the song starts in tempo and in phase. During the song the phase remains fixed, while the music period adapts to the walking period. The subject entrains 


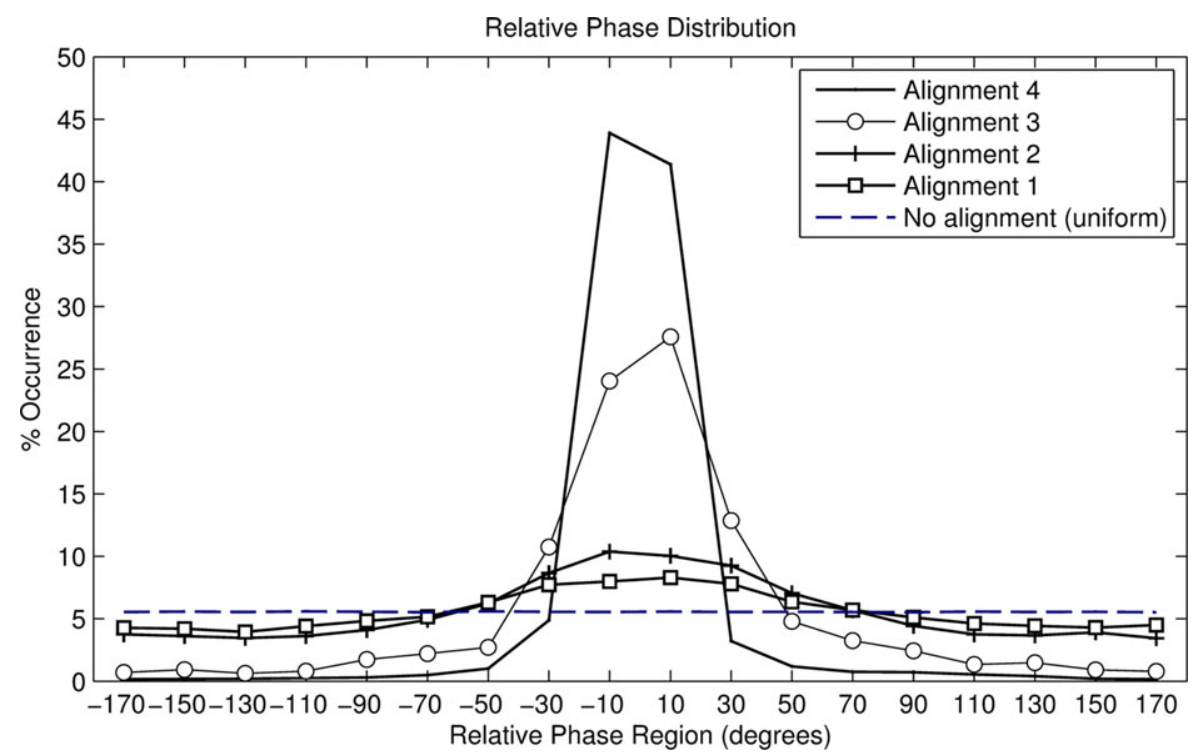

Figure 2. Angular histogram of the four alignment strategies.

to the phase of the song; and (4) the song starts in tempo and in phase. During the song, the period and phase are adapted. The music player follows subjects' changes in period and phase. Each strategy was built on the knowledge and experience learned from experiments in which the alignment strategies were tested in view of maximizing spontaneous synchronization. Table 1 (top) summarizes the alignment strategies and the experimental contexts according to the indicated parameters. The different test conditions prevent from making direct comparisons between the data produced by the experiments. However, we can sift out a number of insights concerning the effect of the strategies on entrainment. Table 1(bottom) summarizes the statistical findings for each experiment, and the angular histogram for each experiment is shown in Figure 2.

\section{Four alignment strategies}

\section{Alignment strategy 1: period adaptive, phase random}

The first alignment strategy matches the tempo of the music (BPM) with the observed tempo of the gait (SPM). The relative phase is neglected, which means that the music starts at whatever time interval is taken between two steps, and the phase of the music is not adapted during the song. The observed gait tempo is based on the average gait period of the last 5 seconds. The strategy allows the participant to adjust the relative phase by taking smaller or bigger steps. However, when this strategy was used, it was observed that the human gait rarely remains constant and that the average frequency continuously fluctuates around a mean. The reason is that music tempo and gait tempo tend to "wobble," that is, alternate around the mean. An in-depth data analysis reveals that participants subsequently try to readjust their pace in an attempt to get back in time with the music. However, the system reacts to this change, and phase alignment is lost. The results suggest that stable spontaneous synchronization would profit from a less adaptive alignment strategy, similar to the findings of Fairhurst et al. ${ }^{8}$

\section{Alignment strategy 2: period fixed, phase random}

In this alignment strategy, the music period holds steady once it has been chosen on the basis of previously observed gait tempo. Similar to the first alignment strategy, the relative phase is not used. When the song ends, a new song is selected on the basis of the most recent measurement of the gait tempo. Experiments reveal that this alignment strategy is slightly better (Table 1) owing to an increased resultant vector length. The in-depth data analysis of individual performances reveals difficulties in the first phase of the entrainment, where the subject 


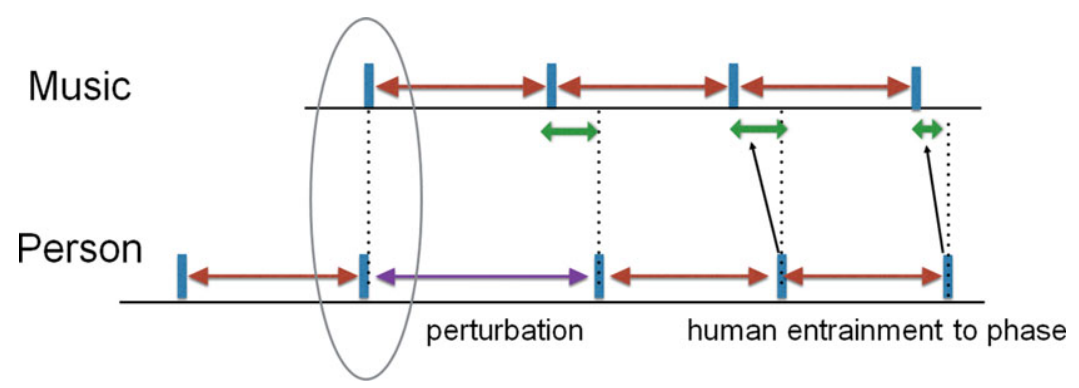

Figure 3. Alignment strategy 3. The beat and footfall are represented by the short vertical lines on a horizontal line that represents time. The double arrows on top of a horizontal line represent period, and those below the horizontal line represent relative phase. The oval shape draws attention to the moment at which the music begins.

tries to find the beat. However, once the beat is found, it is easier to keep it and stay "locked" in phase. Once in-phase synchrony is obtained, the synchronization is usually maintained until the end of the song. We also observed that the initial music tempo selection is often several BPMs off target. During the $5 \mathrm{~s}$ needed for the music player to adapt, the actual gait frequency will most likely change, and the music selection will no longer be optimal. This problem is inherent with real-time step detection, especially when the music player is nonadaptive. Another disadvantage of this alignment strategy is its insensitivity to sudden gait period changes. These occurred frequently in our test owing to environmental factors such as road crossings (although these were kept to a minimum), obstacles such as other pedestrians, or when participants stopped to look for the next directional marker. In such cases, the new gait period rarely matched the gait period before the obstacle occurred, creating a substantial difference between music and gait period and, thus, less synchronization. The tests suggested that improvements could be made by considering an inphase lock at the start of a song and by reintroduction of period adaptation.

\section{Alignment strategy 3: period adaptive, phase starting $0^{\circ}$}

This alignment strategy (Fig. 3) takes the relative phase as well as the period into account. Music is played with an adaptive period based on the average gait period of the last $5 \mathrm{~s}$, which is identical to the first alignment strategy. In addition, this alignment strategy introduces a song start on the basis of the user's gait phase. The song begins playing when the relative phase is equal to the desired starting phase.
Interestingly, the results are remarkably better than those of previous strategies (Table 1). Subjects tend to stay in phase once a low relative phase angle is obtained. Yet, the only difference from alignment strategy 1 is a fixed starting relative phase angle of $0^{\circ}$ that takes place at the start of the song. This alignment appears to be very effective and encourages stable synchronization. The proposed alignment strategy does seem to solve the problem of finding the in-phase alignment that affected the previous alignment strategies. Because there will always be people who are unable to synchronize, the strategy offers the option of going one step further: using both phase adaptation and a period adaptation.

\section{Alignment strategy 4: period adaptive, phase adaptive to $0^{\circ}$}

This alignment strategy adapts the relative phase angle when an allowed deviation from $-30^{\circ}$ to $30^{\circ}$ is exceeded. If this deviation persists, then an extra period adjustment is applied to enable the phase alignment to converge toward the $0^{\circ}$ relative phase angle. The results of our testing illustrate that this alignment strategy is very effective in maintaining synchronization. Steps taken outside the synchronous range are automatically corrected, although, because of the delay caused by the step detection, the correction may take a few seconds to take effect. Interestingly, with this alignment strategy there is basically no human entrainment needed to get in phase. Instead, the entrainment is done by the music player. All that is required of the participant is "being in phase" without "getting in phase," and s/he is not required even to "keep in phase." 


\section{Discussion}

\section{The power of beat locking}

Although the alignment strategies have been tested in various contexts, they can be compared indirectly by considering the in-depth analyses of how strategies influence entrainment. As a consequence, we observed that the manipulation of the relative phase (strategies 3 and 4) makes a significant difference. For example, in strategy 3 the music is always set in-phase at the start of a song. This helps to maintain stable synchronization immediately. When the tempo changes, the phase will also change, but it will remain in synchrony with the participant. Alignment strategy 4 is a case apart because the music's rhythm is now entirely adapted to the person. There is no entrainment required on the part of the person, only alignment. If the person changes phase or period, then the music player will respond immediately, accounting for a short system latency. The observations show that spontaneous human entrainment seems most effective in alignment strategy 3 for reasons that can be attributed to the in-phase lock at the beginning of the song. Interestingly, human entrainment to find the beat can be eliminated and replaced by machine entrainment, as shown in alignment strategy 4 .

\section{Shades of entrainment}

A key observation is that spontaneous entrainment drives the coupled rhythms toward a stable in-phase alignment. Strategies that immediately lock the subject in phase with the music at the start of each song are superior. We assume that they allow the subject to predict the beat more accurately right from the very start of a song. In contrast, strategies that require finding the beat at the beginning of the song present more of a challenge because predictions are less established and phase-correction adjustments may be large and inaccurate. Alignment strategy 4 not only eliminates the human task of finding the beat, it also eliminates the task of keeping the beat. The above findings suggest that a distinction should be made among finding the beat, holding the beat, and being the beat. The difference can be explained by internal models that link motor control with sensory outcomes. Finding the beat is difficult because the gait pattern has a proper movement cycle, which should be changed if it is to become aligned with the beat. The internal model that motors activity with expected sensory outcomes has proven to be unreliable because an accurate timing model of the expected sensory outcome (namely footfall aligned with the beat) is lacking. Holding the beat is less difficult because a more stable association of motor control and expected sensory output (the beat) can be used. Accordingly, less effort may be needed to hold or keep the beat than to find the beat.

\section{Study limitations}

Real-time gait frequency detection has an inherent delay because at least two steps are needed to determine the tempo; but more are required for determining a stable gait tempo. The implied latency imposes some restriction on the alignment strategies. However, because of this latency, part of the variability of the human gait ${ }^{15}$ is averaged, which results in a more stable SPM value. As to making comparisons of our work with the work of others, it should be noted that our emphasis on spontaneous entrainment differs from most of the walkingrunning studies that have been conducted thus far. When participants are requested to synchronize with music played by means of a nonadaptive music player, one typically finds that synchronous music has numerous advantages related to ergogenic, physiological, and/or psychophysiological aspects. ${ }^{16-21}$ This calls on the participant to engage with music in an intentional way, which might alter the participant's natural gait pattern. However, we believe that in many applications the instructed entrainment is not always realistic. We also believe that it is important to understand the subliminal effects of entrainment and alignment, and here too, further research is needed.

\section{Strengths of D-Jogger and open questions}

The above results suggest that D-Jogger can serve as an assistive technology to motivate people to exercise physically. D-Jogger can also be used as an assistive technology in a clinical context (e.g., as a smart Walkman for Parkinson patients), or for physical rehabilitation. Apparently, not everybody can synchronize to music, even after receiving instruction. ${ }^{22-24}$ This group of people can be aided with a system that facilitates human entrainment or cancels it entirely (alignment strategy 4). Because D-Jogger is an entrainment mediator between human rhythm and musical rhythm, it offers perspectives for studying biological rhythms (respiration, heartbeat). 


\section{Conclusions}

The goal of this paper was to study the mediators of entrainment that affect the way in which rhythms influence each other. We demonstrated that different setups (hereafter referred to as alignment strategies) can indeed have an effect on spontaneous human entrainment behavior. We found that entrainment may be less effective if the attractor (zero relative phase) fluctuates (which is often the case in an interactive setup). The solution revealed that the relative phase should be set close to zero right from the start of the interaction. Being in phase at the start seems to dramatically raise the possibility of obtaining a stable synchronization (through keeping the in-phase alignment), as it avoids the effort of finding in phase. From a theoretical point of view, this means that entrainment is most effective when it maintains a given stable alignment between movement and music. Finding this stable alignment is much harder to achieve. The impetus fueling the creation of the D-Jogger was a need to produce a technology that would empower people to walk or run at their own pace using a music player that automatically adapts to their tempo. In addition, there are several applications in the domain of assistive technologies and physical rehabilitation, applications that are even tailored toward people who cannot entrain to an external auditory stimulus. We believe that our technology and the implied results offer interesting perspectives for further study, which include studies in neuroscience and provide useful applications in the areas of sports and physical rehabilitation.

\section{Acknowledgments}

This study has been carried out in the context of the Methusalem project entitled "Embodied music cognition and mediation technologies for cultural and creative applications." The authors wish to thank all partners who made the experiments possible, including all IPEM colleagues at the University of Ghent. D-Jogger is a core technology of the European ICT Project "BeatHealth" (http://www.euromov.eu/beathealth/homepage).

\section{Conflicts of interest}

The authors declare no conflicts of interest.

\section{References}

1. Repp, B.H. \& Y.-H. Su. 2013. Sensorimotor synchronization: a review of recent research (2006-2012). Psychonom. Bull. Rev. 20: 403-452.

2. Haken, H., J.S. Kelso \& H. Bunz. 1985. A theoretical model of phase transitions in human hand movements. Biol. Cybern. 51: 347-356.

3. Kelso, J., J. Del Colle \& G. Schöner. 1990. “Action-perception as a pattern formation process." In Attention and Performance. M.J. Jeannerod, Ed.: 139-169. Hillsdale, NJ: Lawrence Erlbaum Associates.

4. Schmidt, R.C. \& M.J. Richardson. 2008. "Dynamics of interpersonal coordination." In Coordination: Neural, Behavioral and Social Dynamics. A. Fuchs, Ed.: 281-308. Berlin: Springer.

5. Nessler, J.A. \& S.J. Gilliland. 2009. Interpersonal synchronization during side by side treadmill walking is influenced by leg length differential and altered sensory feedback. Hum. Mov. Sci. 28: 772-785.

6. Schmidt, R.C., C. Carello \& M.T. Turvey. 1990. Phase transitions and critical fluctuations in the visual coordination of rhythmic movements between people. J. Exp. Psych. Hum. Percept. Perf. 16: 227.

7. Miyake, Y. 2009. Interpersonal synchronization of body motion and the Walk-Mate walking support robot. IEEE Trans. Robot. 25: 638-644.

8. Fairhurst, M.T., P. Janata \& P.E. Keller. 2013. Being and feeling in sync with an adaptive virtual partner: brain mechanisms underlying dynamic cooperativity. Cereb. Cortex 23: 2592-2600.

9. Moens, B., C. Muller, L. van Noorden, et al. 2014. Encouraging spontaneous synchronisation with D-Jogger, an adaptive music player that aligns movement and music. PLoS One 9: e114234.

10. Pappas, I.P. et al. 2001. A reliable gait phase detection system. IEEE Trans. Neural Syst. Rehabil. Eng. 9: 113-125.

11. Ying, H., C. Silex \& A. Schnitzer. Automatic step detection in the accelerometer signal. IFMBE Proc. 13: 80-85.

12. Moens, B. 2010. Sensor Evaluation for Real-Time Pace and Step Detection for Sonic Interaction. Ghent: Ghent University.

13. Dixon, S. 2007. Evaluation of the audio beat tracking system beatroot. J. New Music Res. 36: 39-50.

14. Elastique time stretching \& pitch shifting SKs. Cited September 2014. http://www.zplane.de/index.php?page= description-elastique.

15. Scafetta, N., D. Marchi \& B.J. West. 2009. Understanding the complexity of human gait dynamics. Chaos 19: 026108.

16. Simpson, S.D. \& C.I. Karageorghis. 2006. The effects of synchronous music on 400-m sprint performance. J. Sports Sci. 24: 1095-1102.

17. Lane, A.M., P.A. Davis \& T.J. Devonport. 2011. Effects of music interventions on emotional states and running performance. JSSM 10: 400.

18. Terry, P.C. et al. 2012. Effects of synchronous music on treadmill running among elite triathletes. J. Sci. Med. Sport 15: 52-57.

19. Bacon, C., T. Myers \& C. Karageorghis. 2012. Effect of musicmovement synchrony on exercise oxygen consumption. J. Sports Med. Phys. Fit. 52: 359-365. 
20. Hoffmann, C.P., G. Torregrosa \& B.G. Bardy. 2012. Sound stabilizes locomotor-respiratory coupling and reduces energy cost. PLoS One 7: e45206.

21. Karageorghis, C.I. \& D.-L. Priest. 2012. Music in the exercise domain: a review and synthesis (Part II). J. Sport Exerc. Psychol. 5: 67-84.

22. Phillips-Silver, J. et al. 2011. Born to dance but beat deaf: a new form of congenital amusia. Neuropsychologia 49: 961969.
23. Styns, F. et al. 2007. Walking on music. Hum. Mov. Sci. 26: 769-785.

24. Leman, M. et al. 2013. Activating and relaxing music entrains the speed of beat synchronized walking. PLoS One 8: e67932.

25. Fisher, N.I. 1995. Statistical Analysis of Circular Data. Cambridge: Cambridge University Press.

26. Wilkie, D. 1983. Rayleigh test for randomness of circular data. Appl. Statist. 32: 311-312. 